\title{
Knowledge and Behaviors towards COVID-19 among University of Aleppo Students: An Online Cross-sectional Survey
}

\author{
Aos Alhamid ${ }^{1}$, Ziad Aljarad $^{2}$, Ahmad Alhamid ${ }^{1}$ \\ ${ }^{1}$ Medical student, Faculty of Medicine, University of Aleppo, Aleppo, Syria \\ ${ }^{2}$ Gastroenterology specialist - PhD, Aleppo University Hospital, Aleppo, Syria
}

Correspondence to Aos Alhamid : Medical student, Faculty of Medicine, University of Aleppo, Aleppo, Syria ; aos.alhamid.98@gmail.com tel: +963 958366842

\section{Abstract}

Background: Coronavirus Disease 2019 (COVID-19) is an infection caused by a novel coronavirus that affects respiratory tract. People's awareness and knowledge of, and behavior and attitude toward COVID-19 are scarcely investigated, making medical literature related to this point poor. we aim to measure the knowledge of, and the reaction to COVID-19 among University of Aleppo students in Syria, and the determinants of their awareness and behavior regarding this disease.

Materials and Methods: This was an online, questionnaire-based cross-sectional study, that was conducted from 21st March to 30 March 2020. We included undergraduate students of the University of Aleppo (Syria). The questionnaire consisted of three sections: Demographics ,knowledge and behaviours . Every participant's knowledge was scored from 0-13 depending on the number of correct answers in the knowledge section. The correctness was judged depending on WHO recommendations at the time of questionnaire administration. P-value of 0.05 was considered significant.

Results: Among this well-educated and predominantly medical and health-related students, 682 (45.4\%) students had a good knowledge level, which is somehow disappointing. The current study shows that 1 st year students and non-medical specialties students and smokers had significantly lower knowledge levels than others. On contrary, residing with less people -which may indicate a higher socioeconomic status-, was associated with a higher knowledge level. We also found that commitment to preventive measures was in general satisfying and correlated significantly with knowledge level and gender in most cases.

Conclusion: Junior students, non-medical specialties, smokers and those who live with high number of people are vulnerable to less knowledge level and awareness campaigns should concentrate on them. Increasing awareness is useful to increase commitment to preventive 
medRxiv preprint doi: https://doi.org/10.1101/2020.07.11.20151035; this version posted July 14, 2020. The copyright holder for this preprint (which was not certified by peer review) is the author/funder, who has granted medRxiv a license to display the preprint in perpetuity. It is made available under a CC-BY-NC-ND 4.0 International license .

measures, and groups that have less adherence to preventive measures, as described in detail, should be taken into consideration while designing public health responses. Finally, we should be aware of the negative impact of quarantine on public health to take it into consideration for current campaigns and future policies.

\section{Introduction}

Coronavirus Disease 2019 (COVID-19) is an infection caused by a novel coronavirus that affects respiratory tract, it was first detected in Wuhan, China, in December 2019. Whole-genome sequencing of the virus declares that it is a betacoronavirus closely related to the Severe Acute Respiratory Syndrome (SARS) virus [1]. The origin of this virus is still questionable [2], but a zoonotic origin from bat and pangolin is suspected [3].

Most symptomatic infected people develop symptoms such as fever, fatigue, Dry cough, Anorexia, Myalgias, Sputum production and dyspnea. And 20\% of patients may develop Acute respiratory distress syndrome (ARDS) [4]. Some studies have also reported gastrointestinal symptoms in $2-10 \%$ of cases such as diarrhea, abdominal pain, and vomiting $[5,6]$ and neurological symptoms in $36.4 \%$ of cases such as disturbed consciousness, headache, and paresthesia [7].

COVID-19 is transmitted from human-to-human through respiratory droplet, feco-oral route, and direct contact, and has an incubation period of 2-14 days [8].

COVID-19 is a rapidly expanding global threat, placing an intolerable burden on healthcare services. Number of cases is dramatically increasing every day. Until 21 March 2020 , 292142 cases were detected worldwide, with 12784 deaths, and on 13 May 2020 4,337,562 cases were detected worldwide, with 292,450 deaths, 1,597,641 recovered cases, and 2,447,471 active cases [9] . It has been considered as a major threat to humans since December 31, 2019 [10] , and lastly defined as a pandemic on 11 March 2020 [11] .

To date, no vaccine or treatment has been approved for this disease, making preventive measures the most effective intervention [11]. This makes the public the main player in combating this pandemic.

People's awareness and knowledge of, and behavior and attitude toward COVID-19 are scarcely investigated, making medical literature related to this point poor.

in this study, we aim to measure the knowledge of, and the reaction to COVID-19 among University of Aleppo students, and the determinants of their awareness and behavior regarding this disease. This study is important to inform governments and concerned organizations about people thoughts and actions, especially the young, and may help to inform the upcoming public health measures and interventions.

\section{Materials and Methods}

We conducted this cross-sectional study from 21st March to 30 March 2020. It was hard to make it a field-based study because of limited access to university students in this epidemic situation and because of the governmental measure to close the university temporally, so we made it a web-based study . 
Therefore, we formed an online questionnaire and shared it with students on faculties Facebook, Telegram, and Whatsapp groups. The post introducing the questionnaire contained a background about the study, its aims, and some notes about filling the questionnaire, as well as the link to the questionnaire. The front page of the questionnaire contained a detailed informed consent statement for participation, and a button through it the participant can confirm the consent. We included all students from the University of Aleppo, from both sexes and all ages who were studying in a bachelor degree or equivalent program, or in technical middle institutes. We excluded participants from other universities and graduated students.

the questionnaire consisted of 3 parts :Demographics , awareness and knowledge of COVID-19, and behaviors toward COVID-19 and preventive measures. The Demographics part consisted of 12 questions about socio-economic and demographic characteristics, while the awareness and knowledge part included 13 questions on symptoms, transmission ways, preventive ways, therapy, and a question on the resource of participants information. Behavioral part contained 10 questions about the participants commitment to preventive measures and the impact of the quarantine on their daily habits.

Questions in knowledge part were true-false-I do not know questions. Participants who answered the question correctly took one point for each question , and who answered incorrectly or said " I don't know " took zero points for the question. The correctness was considered depending on WHO recommendations on the day of questionnaire administration. [12] So, the degrees ranged from 0 to 13.

We conducted statistical analysis using SPSS (Version 22.0; SPSS Inc.: Chicago, IL, USA). Categorical variables were presented as frequencies and percentages, while continuous data was summarized as mean \pm standard deviation (SD). We calculated Odds ratios (OR) with confidence intervals (CI) using logistic regression analysis. Whichever indicated, we used Chisquare, Fisher's exact test and Student T-test tests to calculate the p-values. The p-value less than 0.05 was considered statistically significant.

This study was approved by the research committee of the University of Aleppo.

\section{Results}

Among the 1502 students who responded, 834 (55.5\%) were females, 881 (58.7\%) studied a medical or health-related specialty, and 1423(94.7\%) of them were single. Advanced students (who are in the 3rd year or more) constituted for the majority of the sample (904,60.2\%). We found that $447(29.8 \%)$ students were regular cigarettes or shisha smokers. More details on the demographic and socioeconomic characteristics of the participants are shown in table.1.

Table 1 : characteristics of participants and its frequencies .

original

\begin{tabular}{|l|l|}
\hline Characteristic & Frequency (percentage \%) \\
\hline Gender $(\mathbf{n = 1 5 0 2})$ & \\
\hline
\end{tabular}


medRxiv preprint doi: https://doi.org/10.1101/2020.07.11.20151035; this version posted July 14, 2020. The copyright holder for this preprint (which was not certified by peer review) is the author/funder, who has granted medRxiv a license to display the preprint in perpetuity.

It is made available under a CC-BY-NC-ND 4.0 International license .

\begin{tabular}{|l|c|}
\hline Female & $834(55.5)$ \\
\hline Male & $668(44.5)$ \\
\hline Specialty (n=1502) & $881(58.7)$ \\
\hline Medical & $391(26)$ \\
\hline Engineering & $203(13.5)$ \\
\hline literary & $27(1.8)$ \\
\hline sciences & \\
\hline Year (n=1502) & $344(22.9)$ \\
\hline 1 & $254(16.9)$ \\
\hline 2 & $904(60.2)$ \\
\hline 3 or more & \\
\hline You descend from (n=1502) & $1276(85)$ \\
\hline Urban & $226(15)$ \\
\hline Rural & \\
\hline Marital status (n=1502) & $1423(94.7)$ \\
\hline Single & $39(2.6)$ \\
\hline Engaged & $37(2.5)$ \\
\hline Married & $3(0.2)$ \\
\hline Widow or Divorced & \\
\hline You Reside with (n=1502) & $21(1.4)$ \\
\hline Alone & $1280(85.2)$ \\
\hline Family & $201(13.4)$ \\
\hline Other students & \\
\hline $\begin{array}{l}\text { Do you Work beside your } \\
\text { study (n=1502) }\end{array}$ & $1055(70.2)$ \\
\hline No & $428(28.5)$ \\
\hline Yes & \\
\hline $\begin{array}{l}\text { Do you Smoke cigarette or } \\
\text { shisha (n=1502) }\end{array}$ & \\
\hline No & \\
\hline Yes & \\
\hline & \\
\hline & \\
\hline & \\
\hline
\end{tabular}

Table.2 demonstrates participants' answers to the questions related to the knowledge and awareness of COVID-19. In most questions, the majority of respondents answered correctly to the questions, except for the question K9 $(669,44.6 \%)$ about the need to wear a mask outside, and question $\mathrm{K} 13(573,38.2 \%)$ which was about a rumor that spread virally through social media in Syria reporting a new easy diagnostic method for COVID-10 invented by an unknown 
medRxiv preprint doi: https://doi.org/10.1101/2020.07.11.20151035; this version posted July 14, 2020. The copyright holder for this preprint (which was not certified by peer review) is the author/funder, who has granted medRxiv a license to display the preprint in perpetuity.

It is made available under a CC-BY-NC-ND 4.0 International license .

Japanese scientist. We can also notice that 773 (51.6\%) of participants answered correctly to question $\mathrm{K} 7$ about the possibility of being an asymptomatic COVID-19 patient, making this majority not vast and on borderline. The participants scores ranged from $0 / 13$ to $13 / 13$, with a mean score of COVID-19 knowledge of 9.2干1.8. $123(8.2 \%)$ participants had a poor knowledge level, 697 (46.4\%) had a moderate knowledge level, and 682 (45.4\%) had a good knowledge level. Notably, social media was the main source of information about COVID-19 in 1041 $(69.6 \%)$ of the study sample, while discussions with family and friends was the least common source of information $(373,23.9 \%)$.

Table 2 : participants' answers to the knowledge questions .

original

\begin{tabular}{|c|c|}
\hline Questions & Frequency (percentage \%) \\
\hline \multicolumn{2}{|l|}{$\begin{array}{l}\text { K1. COVID-19 virus is transmitted } \\
\text { through droplets that come out from } \\
\text { the infected person's cough or breath } \\
(\mathbf{n = 1 4 9 4 )}\end{array}$} \\
\hline True $*$ & $1412(94.5)$ \\
\hline False & $50(3.3)$ \\
\hline Don't know & $32(2.1)$ \\
\hline \multicolumn{2}{|l|}{$\begin{array}{l}\text { K2. Young people and people with } \\
\text { normal immunity do not get infected } \\
\text { with the COVID-19 virus } \\
(\mathbf{n = 1 5 0 2})\end{array}$} \\
\hline True & $258(17.2)$ \\
\hline False $*$ & $1157(77)$ \\
\hline Don't know & $87(5.8)$ \\
\hline \multicolumn{2}{|l|}{$\begin{array}{l}\text { K3. There is a current cure for } \\
\text { corona virus } \\
\text { (n=1501) }\end{array}$} \\
\hline True & $79(5.3)$ \\
\hline False * & $1148(76.5)$ \\
\hline Don’t know & $274(18.3)$ \\
\hline \multicolumn{2}{|l|}{$\begin{array}{l}\text { K4. Influenza vaccine can be used as } \\
\text { a vaccine to prevent corona virus } \\
(\mathbf{n = 1 5 0 0 )}\end{array}$} \\
\hline True & $42(2.8)$ \\
\hline False * & $1199(79.9)$ \\
\hline Don't know & $259(17.2)$ \\
\hline K5. Common symptoms of corona & \\
\hline
\end{tabular}


medRxiv preprint doi: https://doi.org/10.1101/2020.07.11.20151035; this version posted July 14, 2020. The copyright holder for this preprint (which was not certified by peer review) is the author/funder, who has granted medRxiv a license to display the preprint in perpetuity.

It is made available under a CC-BY-NC-ND 4.0 International license .

\begin{tabular}{|c|c|}
\hline $\begin{array}{l}\text { virus include fever, dry cough, and } \\
\text { fatigue } \\
(\mathbf{n = 1 4 9 9})\end{array}$ & \\
\hline True * & $1490(99.4)$ \\
\hline False & $1(0.1)$ \\
\hline Don't know & $8(0.5)$ \\
\hline $\begin{array}{l}\text { K6. Sneeze and rhinorrhea is less } \\
\text { common in people with corona than } \\
\text { people with a common cold } \\
(\mathbf{n = 1 5 0 1})\end{array}$ & \\
\hline True * & $958(63.8)$ \\
\hline False & $299(19.9)$ \\
\hline Don't know & $244(16.2)$ \\
\hline $\begin{array}{l}\text { K7. A person can become infected } \\
\text { with the COVID-19 virus without } \\
\text { having fever or shortening their } \\
\text { breathing } \\
(\mathbf{n}=\mathbf{1 4 9 7})\end{array}$ & \\
\hline True * & $773(51.6)$ \\
\hline False & $472(31.5)$ \\
\hline Don't know & $252(16.8)$ \\
\hline $\begin{array}{l}\text { K8.Washing hands with soap and } \\
\text { water is a preventative measure } \\
(\mathbf{n}=\mathbf{1 4 9 9})\end{array}$ & \\
\hline True * & $1478(98.6)$ \\
\hline False & $12(0.8)$ \\
\hline Don’t know & $9(0.6)$ \\
\hline $\begin{array}{l}\text { K9. Everyone must cover their } \\
\text { mouth and nose with a mask when } \\
\text { going outside } \\
(\mathbf{n}=\mathbf{1 4 9 9})\end{array}$ & \\
\hline True & $785(52.4)$ \\
\hline False * & $669(44.6)$ \\
\hline Don't know & $45(3)$ \\
\hline $\begin{array}{l}\text { K10. Avoiding crowded places and } \\
\text { staying at home is a protective } \\
\text { measure } \\
(\mathbf{n = 1 4 9 8})\end{array}$ & \\
\hline True ${ }^{*}$ & $1489(99.4)$ \\
\hline False & $5(0.3)$ \\
\hline
\end{tabular}


medRxiv preprint doi: https://doi.org/10.1101/2020.07.11.20151035; this version posted July 14, 2020. The copyright holder for this preprint (which was not certified by peer review) is the author/funder, who has granted medRxiv a license to display the preprint in perpetuity.

It is made available under a CC-BY-NC-ND 4.0 International license .

\begin{tabular}{|c|c|}
\hline \multirow{2}{*}{$\begin{array}{l}\text { Don't know } \\
\text { K11. The incubation period of the } \\
\text { COVID-19 Virus is } 2 \text { to } 14 \text { days } \\
(\mathbf{n}=\mathbf{1 4 9 7})\end{array}$} & $4(0.3)$ \\
\hline & \\
\hline True * & $1323(88.4)$ \\
\hline False & $70(4.7)$ \\
\hline Don’t know & $104(6.9)$ \\
\hline \multicolumn{2}{|l|}{$\begin{array}{l}\text { K12. Most cases of COVID-19 } \\
\text { eventually heal } \\
(\mathbf{n = 1 4 9 9 )}\end{array}$} \\
\hline True * & $979(65.3)$ \\
\hline False & $266(17.7)$ \\
\hline Don't know & $254(16.9)$ \\
\hline \multicolumn{2}{|l|}{$\begin{array}{l}\text { K13. COVID-19 virus infection can } \\
\text { be diagnosed by holding the breath } \\
\text { for } 20 \text { seconds and then exhaling } \\
\text { quietly.. And when the infection is } \\
\text { present, the cough will provoke } \\
(\mathbf{n}=\mathbf{1 4 9 9})\end{array}$} \\
\hline True & $408(27.2)$ \\
\hline False * & $573(38.2)$ \\
\hline Don’t know & $518(34.6)$ \\
\hline \multicolumn{2}{|l|}{$\begin{array}{l}\text { K14. From where have you got your } \\
\text { knowledge about covid-19 ( more } \\
\text { than one answer is possible ) } \\
(\mathbf{n = 1 4 9 6 )}\end{array}$} \\
\hline Social media & $1041(69.6)$ \\
\hline Television or radio & $418(27.9)$ \\
\hline $\begin{array}{l}\text { Official government websites / } \\
\text { organization and health agencies } \\
\text { website }\end{array}$ & $989(66.1)$ \\
\hline Discussions with family and friends & $373(23.9)$ \\
\hline
\end{tabular}

$$
*=\text { correct answer }
$$

Table. 3 shows the behavior toward COVID-19 as reported by the participating students. We can notice that a considerable percentage of participants are committed to the preventive measures. $712(47.7 \%)$ participants stopped going out to crowded places, 857 (57.2\%) stopped going out with friends, and $896(59.8 \%)$. In addition, as presented in table.3, there are some participants who were not used to do these behaviors in the first place, even before the pandemic. Most 
medRxiv preprint doi: https://doi.org/10.1101/2020.07.11.20151035; this version posted July 14, 2020. The copyright holder for this preprint (which was not certified by peer review) is the author/funder, who has granted medRxiv a license to display the preprint in perpetuity.

It is made available under a CC-BY-NC-ND 4.0 International license .

respondents had good hand-washing practices: 1380 (92.1\%) students wash their hands more than 3 times a day, and $1323(88.3 \%)$ students use soap in all their hand-washes. Only 109 $(7.3 \%)$ always wear a mask when going out, while the immense majority did not (1120, 75.1\%). $825(55.3 \%)$ of participants sometimes keep a safe distance from others, while, unfortunately, $415(27.8 \%)$ of them do not. Interestingly, $692(46.1 \%)$ and $1323(88.3 \%)$ of the study sample increased their consumption of food and internet, respectively, during the quarantine. It is also notable that 95 (21.3\%) of smokers had higher smoking rate since the beginning of quarantine .

Table. 3 : participants' answers to the behaviors questions .

original

\begin{tabular}{|c|c|}
\hline Questions & Frequency (percentage \%) \\
\hline \multicolumn{2}{|l|}{$\begin{array}{l}\text { B1. Do you still go out to crowded } \\
\text { places (such as markets, parks, and } \\
\text { public squares)? } \\
(\mathbf{n = 1 4 9 4 )}\end{array}$} \\
\hline Yes, as in the past & $18(1.2)$ \\
\hline $\begin{array}{l}\text { Yes, less than in the past, but } \\
\text { sometimes without necessity }\end{array}$ & $126(8.4)$ \\
\hline Yes, just for necessity & $477(31.9)$ \\
\hline $\begin{array}{l}\text { I stopped going out to crowded } \\
\text { places }\end{array}$ & $712(47.7)$ \\
\hline $\begin{array}{l}\text { I primarily do not go out to } \\
\text { crowded places }\end{array}$ & $161(10.8)$ \\
\hline \multicolumn{2}{|l|}{$\begin{array}{l}\text { B2. Do you still go out with your } \\
\text { friends? } \\
(\mathbf{n}=\mathbf{1 4 9 9})\end{array}$} \\
\hline Yes, as in the past & $46(3.1)$ \\
\hline $\begin{array}{l}\text { Yes, less than in the past, but } \\
\text { sometimes without necessity }\end{array}$ & $229(15.2)$ \\
\hline Yes, just for necessity & $306(20.4)$ \\
\hline I stopped going out with friends & $857(57.2)$ \\
\hline $\begin{array}{l}\text { I primarily do not go out with } \\
\text { friends }\end{array}$ & $61(4.1)$ \\
\hline \multicolumn{2}{|l|}{$\begin{array}{l}\text { B3. Do you still eat fast food? } \\
(\mathbf{n}=\mathbf{1 4 9 9})\end{array}$} \\
\hline Yes, as in the past & $59(3.9)$ \\
\hline $\begin{array}{l}\text { Yes, less than in the past, but } \\
\text { sometimes without necessity }\end{array}$ & $123(8.2)$ \\
\hline Yes, just for necessity & $251(16.7)$ \\
\hline I stopped eating fast food & $896(59.8)$ \\
\hline I primarily do not eat fast food & $170(11.3)$ \\
\hline
\end{tabular}


medRxiv preprint doi: https://doi.org/10.1101/2020.07.11.20151035; this version posted July 14, 2020. The copyright holder for this preprint (which was not certified by peer review) is the author/funder, who has granted medRxiv a license to display the preprint in perpetuity.

It is made available under a CC-BY-NC-ND 4.0 International license .

\begin{tabular}{|c|c|}
\hline $\begin{array}{l}\text { B4. How often do you wash your } \\
\text { hands daily? } \\
(\mathbf{n}=\mathbf{1 4 9 8})\end{array}$ & \\
\hline never & $2(0.1)$ \\
\hline $1-3$ & $116(7.7)$ \\
\hline More than 3 & $1380(92.1)$ \\
\hline $\begin{array}{l}\text { B5. Do you always use soap when } \\
\text { you wash your hands? } \\
(\mathbf{n}=\mathbf{1 4 9 8})\end{array}$ & \\
\hline Always & $1323(88.3)$ \\
\hline Not always & $175(11.7)$ \\
\hline $\begin{array}{l}\text { B6. Do you wear a mask when you } \\
\text { go out on the street? } \\
(\mathbf{n = 1 4 9 2})\end{array}$ & \\
\hline Always & $109(7.3)$ \\
\hline Sometimes & $263(17.6)$ \\
\hline never & $1120(75.1)$ \\
\hline $\begin{array}{l}\text { B7. Do you keep a safe distance of } \\
\text { more than a meter when meeting } \\
\text { anyone? } \\
(\mathbf{n = 1 4 9 1 )}\end{array}$ & \\
\hline Always & $251(16.8)$ \\
\hline Sometimes & $825(55.3)$ \\
\hline never & $415(27.8)$ \\
\hline $\begin{array}{l}\text { B8. Has your consumption of food } \\
\text { increased since the beginning of } \\
\text { the home quarantine? } \\
(\mathbf{n}=\mathbf{1 4 9 5})\end{array}$ & \\
\hline Yes & $692(46.1)$ \\
\hline No & $803(53.7)$ \\
\hline $\begin{array}{l}\text { B9. Has the number of hours spent } \\
\text { on the Internet increased since the } \\
\text { beginning of the home quarantine } \\
? \\
(\mathbf{n}=\mathbf{1 4 9 9})\end{array}$ & \\
\hline Yes & $1323(88.3)$ \\
\hline No & $176(11.7)$ \\
\hline $\begin{array}{l}\text { B10. If you smoke a cigarette or } \\
\text { shisha, have you increased your } \\
\text { consumption of cigarettes or shisha }\end{array}$ & \\
\hline
\end{tabular}




\begin{tabular}{|l|c|}
\hline $\begin{array}{l}\text { since the beginning of the home } \\
\text { quarantine? } \\
(\mathbf{n = 4 4 5 )}\end{array}$ \\
\hline Yes & $95(21.3)$ \\
\hline No & $350(78.7)$ \\
\hline
\end{tabular}

Table.4 shows the correlation between knowledge level and different variables. Specialty, year of study, who the student resides with, working status and smoking status were significantly associated with knowledge level.

Table. 4 : the correlation between variables and knowledge level .

original

\begin{tabular}{|c|c|c|c|}
\hline & \multicolumn{3}{|c|}{ Knowledge level } \\
\hline & $\begin{array}{l}\text { Poor } \\
\mathrm{n}(\%)\end{array}$ & $\begin{array}{c}\text { Moderate } \\
\text { n (\%) }\end{array}$ & $\begin{array}{l}\text { Good } \\
\mathrm{n}(\%)\end{array}$ \\
\hline \multicolumn{4}{|l|}{ Gender } \\
\hline female & $68(55.3)$ & $407(58.4)$ & $359(52.6)$ \\
\hline male & $55(44.7)$ & $290(41.6)$ & $323(47.4)$ \\
\hline \multicolumn{4}{|l|}{ Specialty * } \\
\hline Medical & $29(23.6)$ & $322(46.2)$ & $530(77.7)$ \\
\hline Non-medical & $94(76.4)$ & $375(53.8)$ & $152(22.3)$ \\
\hline \multicolumn{4}{|l|}{ Year * } \\
\hline 1 & $49(39.8)$ & $204(29.3)$ & $91(13.3)$ \\
\hline 2 & $22(17.9)$ & $132(18.9)$ & $100(14.7)$ \\
\hline 3 or more & $52(42.3)$ & $361(51.8)$ & $491(72)$ \\
\hline \multicolumn{4}{|l|}{ Descent } \\
\hline Rural & $14(11.4)$ & $116(16.6)$ & $96(14.1)$ \\
\hline Urban & $109(88.6)$ & $581(83.4)$ & $586(85.9)$ \\
\hline \multicolumn{4}{|l|}{ Marital status } \\
\hline Single & $120(97.6)$ & $676(97.0)$ & $666(97.7)$ \\
\hline Married and others & $3(2.4)$ & $21(3.0)$ & $16(2.3)$ \\
\hline \multicolumn{4}{|l|}{ Reside with * } \\
\hline Alone & $1(0.8)$ & $10(1.4)$ & $10(1.5)$ \\
\hline Family & $108(87.8)$ & $572(82.1)$ & $600(88)$ \\
\hline With other friends & $14(11.4)$ & $115(16.5)$ & $72(10.6)$ \\
\hline \multicolumn{4}{|l|}{ Working * } \\
\hline work & $45(36.6)$ & $205(29.4)$ & $178(26.1)$ \\
\hline Don't work & $78(63.4)$ & $492(70.6)$ & $504(73.9)$ \\
\hline \multicolumn{4}{|l|}{ Smoking * } \\
\hline smoke & $49(39.8)$ & $230(33.0)$ & $168(24.6)$ \\
\hline Don't smoke & $74(60.2)$ & $467(67.0)$ & $514(75.4)$ \\
\hline
\end{tabular}


medRxiv preprint doi: https://doi.org/10.1101/2020.07.11.20151035; this version posted July 14, 2020. The copyright holder for this preprint (which was not certified by peer review) is the author/funder, who has granted medRxiv a license to display the preprint in perpetuity.

It is made available under a CC-BY-NC-ND 4.0 International license .

\begin{tabular}{|c|c|c|c|}
\hline $\begin{array}{l}\text { Number Resident } \\
\text { partners }\end{array}$ & & & \\
\hline 5 or less & $95(77.2)$ & $573(82.2)$ & $576(84.5)$ \\
\hline$>5$ & $28(22.8)$ & $124(17.8)$ & $106(15.5)$ \\
\hline Age $*^{*}$ & & & 21.2 \\
\hline Mean & 20.6 & 20.9 & 1.9 \\
\hline Std. Deviation & 2.1 & 2.2 & \\
\hline
\end{tabular}

$* \mathrm{P}<0.05$

table.5 reports the OR and $95 \% \mathrm{CI}$ of the significantly related variables with knowledge level. Specialty, year of study, who you reside with, working, smoking and number of residency partners were significantly associated with knowledge level, depending on P-value or OR(95\% CI).

Table.5 : OR and 95\% CI of the significantly related variables with knowledge level. original

\begin{tabular}{|c|c|}
\hline Variable & OR $(95 \%$ CI $)$ \\
\hline \multicolumn{2}{|l|}{ Moderate vs. poor knowledge level } \\
\hline Specialty (medical vs. non-medical) & $2.783(1.789-4.331)$ \\
\hline Year of study $\left(1^{\text {st }}\right.$ vs. $3^{\text {rd }}$ or more $)$ & $0.600(0.392-0.918)$ \\
\hline Year of study ( $2^{\text {nd }}$ vs. $3^{\text {rd }}$ or more $)$ & $0.864(0.505-1.478)$ \\
\hline $\begin{array}{l}\text { Who do you reside with (Alone vs. with other } \\
\text { students) }\end{array}$ & $1.217(0.145-10.236)$ \\
\hline $\begin{array}{l}\text { Who do you reside with (Family vs. with other } \\
\text { students) }\end{array}$ & $0.645(0.357-1.165)$ \\
\hline Do you Work beside your study (no vs. yes) & $1.385(0.927-2.069)$ \\
\hline Do you smoke (no vs. yes) & $1.344(0.907-1.994)$ \\
\hline $\begin{array}{l}\text { How many resident partners do you have ( } 5 \text { or } \\
\text { less vs. more than } 5 \text { ) }\end{array}$ & $1.362(0.856-2.166)$ \\
\hline \multicolumn{2}{|l|}{ Good vs. poor knowledge level } \\
\hline Specialty (medical vs. non-medical) & $11.302(7.180-17.791)$ \\
\hline Year of study $\left(1^{\text {st }}\right.$ vs. $3^{\text {rd }}$ or more $)$ & $0.197(0.125-0.308)$ \\
\hline Year of study $\left(2^{\text {nd }}\right.$ vs. $3^{\text {rd }}$ or more $)$ & $0.481(0.280-0.828)$ \\
\hline $\begin{array}{l}\text { Who do you reside with (Alone vs. with other } \\
\text { students) }\end{array}$ & $1.944(0.230-16.425)$ \\
\hline $\begin{array}{l}\text { Who do you reside with (Family vs. with other } \\
\text { students) }\end{array}$ & $1.080(0.588-1.984)$ \\
\hline Do you Work beside your study (no vs. yes) & $1.634(1.090-2.449)$ \\
\hline Do you smoke (no vs. yes) & $2.026(1.357-3.025)$ \\
\hline $\begin{array}{l}\text { How many resident partners do you have ( } 5 \text { or } \\
\text { less vs. more than } 5 \text { ) }\end{array}$ & $1.602(1.001-2.562)$ \\
\hline
\end{tabular}


Table.6 demonstrates the association between each behavior and different variables, and table.7reports the OR and $95 \%$ CI of the significantly related variables with each behavior. Statistical significance was concluded evaluated by P-value or OR (95\% CI). In general, gender, specialty and knowledge level were significant determinants of most behaviors. More details can be found in table.7.

Table. 6 : the correlation between variables and behaviors .

original

\begin{tabular}{|c|c|c|c|c|c|c|}
\hline & \multicolumn{2}{|c|}{ B1 } & \multicolumn{2}{|c|}{$\mathrm{B} 2$} & \multicolumn{2}{|c|}{ B3 } \\
\hline & $\begin{array}{l}\text { Stopped } \\
\text { n }(\%)\end{array}$ & $\begin{array}{c}\text { have not } \\
\text { stopped } \\
\text { n (\%) }\end{array}$ & $\begin{array}{l}\text { Stopped } \\
\mathrm{n}(\%)\end{array}$ & $\begin{array}{c}\text { have not } \\
\text { stopped } \\
\text { n (\%) }\end{array}$ & $\begin{array}{l}\text { Stopped } \\
\mathrm{n}(\%)\end{array}$ & $\begin{array}{c}\text { have not } \\
\text { stopped } \\
\text { n (\%) }\end{array}$ \\
\hline \multicolumn{7}{|l|}{ Gender } \\
\hline female & $506(71.1)$ & $237(38.2)$ & $628(73.3)$ & $169(29.1)$ & $536(59.8)$ & $216(49.9)$ \\
\hline male & $206(28.9)$ & $\begin{array}{c}384(61.8) \\
*\end{array}$ & $229(26.7)$ & $\begin{array}{c}412 \\
(70.9)^{*}\end{array}$ & $360(40.2)$ & $\begin{array}{c}217 \\
(50.1)^{*}\end{array}$ \\
\hline \multicolumn{7}{|l|}{ Specialty } \\
\hline Medical & $422(59.3)$ & $359(57.8)$ & $522(60.9)$ & $319(54.9)$ & $519(57.9)$ & $265(61.2)$ \\
\hline Non-medical & $290(40.7)$ & $262(42.2)$ & $335(39.1)$ & $\begin{array}{c}262 \\
(45.1)^{*}\end{array}$ & $377(42.1)$ & $168(38.8)$ \\
\hline \multicolumn{7}{|l|}{ Year } \\
\hline 1 & $161(22.6)$ & $131(21.1)$ & $202(23.6)$ & $125(21.5)$ & $198(22.1)$ & $88(20.3)$ \\
\hline 2 & $121(17.0)$ & $100(16.1)$ & $140(16.3)$ & $103(17.7)$ & $143(16.0)$ & $89(20.6)$ \\
\hline 3 or more & $430(60.4)$ & $390(62.8)$ & $515(60.1)$ & $353(60.8)$ & 555 (61.9) & $256(59.1)$ \\
\hline \multicolumn{7}{|l|}{ Descent } \\
\hline Rural & $97(13.6)$ & $99(15.9)$ & $108(12.6)$ & $105(18.1)$ & $138(15.4)$ & $52(12.0)$ \\
\hline Urban & $615(86.4)$ & $522(84.1)$ & $749(87.4)$ & $\begin{array}{c}476 \\
(81.9)^{*}\end{array}$ & $758(84.6)$ & $381(88.0)$ \\
\hline \multicolumn{7}{|l|}{ Marital status } \\
\hline Single & $696(97.8)$ & $602(96.9)$ & $839(97.9)$ & $562(96.7)$ & $876(97.8)$ & $416(96.1)$ \\
\hline $\begin{array}{c}\text { Married and } \\
\text { others }\end{array}$ & $16(2.2)$ & $19(3.1)$ & $18(2.1)$ & $19(3.3)$ & $20(2.2)$ & $17(3.9)$ \\
\hline \multicolumn{7}{|l|}{ Reside with } \\
\hline Alone & $11(1.5)$ & $9(1.4)$ & $13(1.5)$ & $8(1.4)$ & $16(1.8)$ & $4(0.9)$ \\
\hline Family & $614(86.2)$ & $525(84.5)$ & $750(87.5)$ & $471(81.1)$ & $763(85.2)$ & $371(85.7)$ \\
\hline $\begin{array}{c}\text { With other } \\
\text { friends }\end{array}$ & $87(12.2)$ & $87(14.0)$ & $94(11.0)$ & $\begin{array}{c}102 \\
(17.6) *\end{array}$ & $117(13.1)$ & $58(13.4)$ \\
\hline \multicolumn{7}{|l|}{ Working } \\
\hline work & $163(22.9)$ & $234(37.7)$ & $198(23.1)$ & $214(36.8)$ & $254(28.3)$ & $124(28.6)$ \\
\hline Don't work & $549(77.1)$ & $\begin{array}{c}387 \\
(62.3)^{*}\end{array}$ & 659 (76.9) & $\begin{array}{c}367 \\
(63.2)^{*}\end{array}$ & $642(71.7)$ & $309(71.4)$ \\
\hline Smoking & & & & & & \\
\hline
\end{tabular}


medRxiv preprint doi: https://doi.org/10.1101/2020.07.11.20151035; this version posted July 14, 2020. The copyright holder for this preprint (which was not certified by peer review) is the author/funder, who has granted medRxiv a license to display the preprint in perpetuity. It is made available under a CC-BY-NC-ND 4.0 International license .

\begin{tabular}{|c|c|c|c|c|c|c|}
\hline smoke & $164(23.0)$ & $244(39.3)$ & $164(19.1)$ & $275(47.3)$ & $248(27.7)$ & $165(38.1)$ \\
\hline Don't smoke & $548(77.0)$ & $\begin{array}{c}377 \\
(60.7)^{*}\end{array}$ & $693(80.9)$ & $\begin{array}{c}306 \\
(52.7)^{*}\end{array}$ & $684(72.3)$ & $\begin{array}{c}268 \\
(61.9)^{*}\end{array}$ \\
\hline $\begin{array}{l}\text { Number of } \\
\text { Resident } \\
\text { partners }\end{array}$ & & & & & \\
\hline 5 or less & $580(81.5)$ & $524(84.4)$ & $704(82.1)$ & $489(84.2)$ & $753(84.0)$ & $359(82.9)$ \\
\hline$>5$ & $132(18.5)$ & $97(15.6)$ & $153(17.9)$ & $92(15.8)$ & $143(16.0)$ & $74(17.1)$ \\
\hline Age & & & & & & 21.0 \\
\hline Mean & 20.9 & 21.2 & 20.8 & 21.3 & 2.2 & 2.3 \\
\hline Std. Deviation & 2.0 & $2.1 *$ & 1.9 & $2.2 *$ & 2.0 & \\
\hline Knowledge level & & & & & & \\
\hline Poor & $46(6.5)$ & $58(9.3)$ & $68(7.9)$ & $45(7.7)$ & $74(8.3)$ & $31(7.2)$ \\
\hline Moderate & $332(46.6)$ & $299(48.1)$ & $387(45.2)$ & $285(49.1)$ & $406(45.3)$ & $219(50.6)$ \\
\hline good & $334(46.9)$ & $264(42.5)$ & $402(46.9)$ & $251(43.2)$ & $416(46.4)$ & $183(42.3)$ \\
\hline
\end{tabular}

$* \mathrm{P}<0.05$

Table.7 : OR and 95\% CI of the significantly related variables with behaviors .

original

\begin{tabular}{|c|c|}
\hline Variable & OR $(95 \% \mathrm{CI})$ \\
\hline \multicolumn{2}{|l|}{ B1 ( stopped vs. have not stopped ) } \\
\hline Gender ( female vs. male ) & $3.980(3.165-5.004)$ \\
\hline Do you Work beside your study (yes vs. no) & $0.491(0.387-0.623)$ \\
\hline Do you smoke (yes vs. no) & $0.462(0.365-0.586)$ \\
\hline Knowledge level (moderate vs. poor) & $1.400(0.922-2.125)$ \\
\hline Knowledge level (good vs. poor) & $1.595(1.049-2.426)$ \\
\hline \multicolumn{2}{|l|}{ B2 ( stopped vs. have not stopped ) } \\
\hline Gender ( female vs. male ) & $6.686(5.289-8.452)$ \\
\hline Specialty (medical vs. non-medical) & $1.280(1.034-1.584)$ \\
\hline You descend from ( urban vs. rural ) & $1.530(1.142-2.049)$ \\
\hline $\begin{array}{l}\text { Who do you reside with (Alone vs. with other } \\
\text { students) }\end{array}$ & $1.763(0.700-4.443)$ \\
\hline $\begin{array}{l}\text { Who do you reside with (Family vs. with other } \\
\text { students) }\end{array}$ & $1.728(1.276-2.339)$ \\
\hline Do you Work beside your study (yes vs. no) & $0.515(0.409-0.650)$ \\
\hline Do you smoke (yes vs. no) & $0.263(0.208-0.333)$ \\
\hline \multicolumn{2}{|l|}{ B3 ( stopped vs. have not stopped ) } \\
\hline Gender ( female vs. male ) & $1.496(1.187-1.884)$ \\
\hline Do you smoke (yes vs. no) & $0.622(0.488-0.793)$ \\
\hline \multicolumn{2}{|l|}{ B4 ( more than 3 vs. 3 or less ) } \\
\hline Gender ( female vs. male ) & $2.737(1.838-4.075)$ \\
\hline Year of study $\left(1^{\text {st }}\right.$ vs. $3^{\text {rd }}$ or more $)$ & $0.589(0.379-0.915)$ \\
\hline Year of study $\left(2^{\text {nd }}\right.$ vs. $3^{\text {rd }}$ or more $)$ & $0.591(0.363-0.961)$ \\
\hline
\end{tabular}




\begin{tabular}{|c|c|}
\hline Do you smoke (yes vs. no) & $0.591(0.402-(0.869)$ \\
\hline Knowledge level (moderate vs. poor) & $2.114(1.221-3.658)$ \\
\hline Knowledge level (good vs. poor) & $3.228(1.811-5.754)$ \\
\hline \multicolumn{2}{|l|}{ B5 (No vs. Yes) } \\
\hline Gender ( female vs. male ) & $0.382(0.275-0.531)$ \\
\hline Specialty (medical vs. non-medical) & $0.646(0.471-0.886)$ \\
\hline You descend from ( urban vs. rural ) & $0.597(0.404-0.883)$ \\
\hline Do you Work beside your study (yes vs. no) & $1.620(1.166-2.250)$ \\
\hline Do you smoke (yes vs. no) & $1.503(1.082-2.086)$ \\
\hline Knowledge level (moderate vs. poor) & $0.535(0.329-0.872)$ \\
\hline Knowledge level (good vs. poor) & $0.363(0.219-0.603)$ \\
\hline \multicolumn{2}{|l|}{ B6 (always vs. never) } \\
\hline Gender ( female vs. male ) & $1.819(1.197-2.766)$ \\
\hline Specialty (medical vs. non-medical) & $0.445(0.299-0.663)$ \\
\hline Do you Work beside your study (yes vs. no) & $0.796(0.520-1.219)$ \\
\hline Knowledge level (moderate vs. poor) & $2.682(1.352-5.319)$ \\
\hline Knowledge level (good vs. poor) & $2.241(1.441-3.485)$ \\
\hline \multicolumn{2}{|l|}{ B6 (sometimes vs. never) } \\
\hline Gender ( female vs. male ) & $1.142(0.871-1.498)$ \\
\hline Specialty (medical vs. non-medical) & $0.633(0.483-0.829)$ \\
\hline Do you Work beside your study (yes vs. no) & $0.638(0.480-0.848)$ \\
\hline Knowledge level (moderate vs. poor) & $1.550(0.931-2.579)$ \\
\hline Knowledge level (good vs. poor) & $1.624(1.221-2.159)$ \\
\hline \multicolumn{2}{|l|}{ B7 (always vs. never) } \\
\hline Gender ( female vs. male ) & $2.092(1.517-2.883)$ \\
\hline You descend from ( urban vs. rural ) & $0.782(0.514-1.191)$ \\
\hline Do you smoke (yes vs. no) & $1.064(0.764-1.483)$ \\
\hline $\begin{array}{l}\text { How many resident partners do you have ( } 5 \text { or } \\
\text { less vs. more than } 5 \text { ) }\end{array}$ & $1.199(0.806-1.784)$ \\
\hline Knowledge level (moderate vs. poor) & $0.624(0.351-1.111)$ \\
\hline Knowledge level (good vs. poor) & $1.139(0.819-1.584)$ \\
\hline Year of study $\left(1^{\text {st }}\right.$ vs. $3^{\text {rd }}$ or more $)$ & $0.771(0.525-1.133)$ \\
\hline Year of study ( $2^{\text {nd }}$ vs. $3^{\text {rd }}$ or more $)$ & $1.116(0.736-1.693)$ \\
\hline \multicolumn{2}{|l|}{ B7 (sometimes vs. never) } \\
\hline Gender ( female vs. male ) & $1.730(1.364-2.195)$ \\
\hline You descend from ( urban vs. rural ) & $0.634(0.461-0.871)$ \\
\hline Do you smoke (yes vs. no) & $1.446(1.121-1.865)$ \\
\hline $\begin{array}{l}\text { How many resident partners do you have ( } 5 \text { or } \\
\text { less vs. more than } 5 \text { ) }\end{array}$ & $1.536(1.134-2.081)$ \\
\hline Knowledge level (moderate vs. poor) & $0.481(0.314-0.738)$ \\
\hline Knowledge level (good vs. poor) & $1.092(0.851-1.401)$ \\
\hline Year of study $\left(1^{\text {st }}\right.$ vs. $3^{\text {rd }}$ or more $)$ & $0.705(0.531-0.937)$ \\
\hline Year of study $\left(2^{\text {nd }}\right.$ vs. $3^{\text {rd }}$ or more $)$ & $0.843(0.608-1.168)$ \\
\hline \multicolumn{2}{|l|}{ B8 ( yes vs. no ) } \\
\hline Gender ( female vs. male ) & $1.574(1.280-1.935)$ \\
\hline
\end{tabular}




\begin{tabular}{|l|l|}
\hline Marital status (single vs. married and other ) & $2.047(1.033-4.058)$ \\
\hline $\begin{array}{l}\text { Who do you reside with (Alone vs. with other } \\
\text { students) }\end{array}$ & $1.148(0.463-2.846)$ \\
\hline $\begin{array}{l}\text { Who do you reside with (Family vs. with other } \\
\text { students) }\end{array}$ & $0.703(0.521-0.947)$ \\
\hline B9 ( yes vs. no ) & $1.510(1.101-2.070)$ \\
\hline Gender ( female vs. male ) & $1.662(1.125-2.457)$ \\
\hline You descend from ( urban vs. rural ) & $3.384(1.688-6.784)$ \\
\hline Marital status (single vs. married and other) & $1.375(0.810-2.334)$ \\
\hline Knowledge level (moderate vs. poor) & $1.736(1.012-2.980)$ \\
\hline Knowledge level (good vs. poor) & $0.541(0.322-0.907)$ \\
\hline B10 ( yes vs. no ) & $0.374(0.195-0.720)$ \\
\hline Gender ( female vs. male ) & $0.292(0.145-0.588)$ \\
\hline Knowledge level (moderate vs. poor) & \\
\hline Knowledge level (good vs. poor) & \\
\hline
\end{tabular}

\section{Discussion:}

To the best of our knowledge, this is the first study to measure awareness of and behaviors and attitudes toward COVID-19 pandemic in Syria. Among this well-educated and predominantly medical and health-related students, 682 (45.4\%) students had a good knowledge level, which is somehow disappointing. The current study shows that $1^{\text {st }}$ year students and non-medical specialties students and smokers had lower knowledge levels than others, which strongly suggests to concentrate on these groups in awareness campaigns. The lower knowledge level among the first mentioned two groups may be explained by the less medical education they received compared to advanced or medical students. The lower knowledge level among smokers seems to be an association with lower health awareness or less interest in personal and public health. On contrary, residing with less people -which may indicate a higher socioeconomic status-, was associated with a higher knowledge level.

Regarding adherence to preventive measures, we can notice that always females higher commitment levels than males. Knowledge level was associated with behavior in most but not all aspects, and behavior was also correlated with the same factors that were found correlated with knowledge, like scholar year, specialty, work, urban or rural descent, smoking and who and how many you reside with. This indicates that higher awareness leads in general to better action.

The quarantine has increased the consumption of food, internet in $692(46.1 \%), 1323(88.3 \%)$ of participants respectively, and increased tobacco consumption in 95 (21.3\%) of smokers, which means that the quarantine may lead to future side effects on public health.

Unfortunately, to our knowledge, there is no peer-reviewed reports on the same topic of our study published from neighboring countries. However, we found a similar Chinese study performed on public, that reported a high knowledge level with a correct answer rates of 70.2- 
medRxiv preprint doi: https://doi.org/10.1101/2020.07.11.20151035; this version posted July 14, 2020. The copyright holder for this preprint (which was not certified by peer review) is the author/funder, who has granted medRxiv a license to display the preprint in perpetuity. It is made available under a CC-BY-NC-ND 4.0 International license .

98.6\%. [13], This might be because China was the first country to be affected with COVID-19, and much more than Syria.

Our study has major limitations. First, the questionnaire is delivered online, which makes quality control challenging, and reduces the accessibility of some vulnerable groups less probable. In addition, our tools were not well developed and standardized because of limited time and accessibility to the target population.

\section{Conclusion:}

In summary, our study reports a somehow low knowledge level of COVID-19 if we took into consideration that the target population was university students. Junior students, non-medical specialties, smokers and those who live with high number of people are vulnerable to less knowledge level and awareness campaigns should concentrate on them. Increasing awareness is useful to increase commitment to preventive measures, and groups that have less adherence to preventive measures, as described in detail, should be taken into consideration while designing public health responses. Finally, we should be aware of the negative impact of quarantine on public health to take it into consideration for current campaigns and future policies.

\section{Data Availability}

Data are available upon request by contacting the corresponding author .

\section{Conflicts of Interest}

The authors declare that there is no conflict of interest regarding the publication of this paper.

\section{Funding Statement}

No funding 
medRxiv preprint doi: https://doi.org/10.1101/2020.07.11.20151035; this version posted July 14, 2020. The copyright holder for this preprint (which was not certified by peer review) is the author/funder, who has granted medRxiv a license to display the preprint in perpetuity.

It is made available under a CC-BY-NC-ND 4.0 International license .

\section{References}

1- Team NCPERE. Vital surveillances: the epidemiological characteristics of an outbreak of 2019 novel coronavirus diseases (COVID-19) - China. China CDC Weekly. 2020;2(8):113-22.

2- World Health Organization (WHO). Coronavirus disease 2019 (COVID-19): situation report-25, 14 February 2020. Available from:https://www.who.int/docs/defaultsource/coronaviruse/situation-reports/20200214-sitrep-25covid-19.pdf

3- Lam TTY, Shum MHH, Zhu HC et al. Identification of 2019-nCoV related coronaviruses in Malayan pangolins in southern China. bioRxiv preprint first posted online February 18, 2020. 2020:2020.2002.2013.945485. doi: 10.1101/2020.02.13.945485. Accessed February 20, 2020.

4- Wang D, Hu B, Hu C, et al. , Clinical Characteristics of 138 Hospitalized Patients With 2019 Novel Coronavirus-Infected Pneumonia in Wuhan, China. UPTODATE 2020. https://www.uptodate.com/contents/coronavirus-disease-2019-covid-19/abstract/36

5- Wang D , Hu B , Hu C, et al. Clinical characteristics of 138 hospitalized patients with 2019 novel coronavirus-infected pneumonia in Wuhan, China. JAMA. 2020; (published online Feb 7.) DOI:10.1001/jama.2020.1585

6- Chen N, Zhou M, Dong X, et al. Epidemiological and clinical characteristics of 99 cases of 2019 novel coronavirus pneumonia in Wuhan, China: a descriptive study. Lancet. 2020; 395: 507-513

7- Mao L., Wang M.D., Chen S.H., et al. , 2020. Neurological manifestations of hospitalized patients with COVID-19 in Wuhan, China: a retrospective case series study. MedRxiv 2020.02.22.20026500.

8- World Health Organization. Novel coronavirus (COVID-19) situation. Available online: https:// https://experience.arcgis.com/experience/685d0ace521648f8a5beeeee1b9125cd (Accessed on 13 March 2020).

9- https://www.worldometers.info/coronavirus/

10- World Health Organization (WHO). Coronavirus disease (COVID-19) outbreak. Available from: https://www.who.int/emergencies/diseases/novel-coronavirus-2019.

11- World health organization. Rolling updates on coronavirus disease (COVID-19). Available from : https://www.who.int/emergencies/diseases/novel-coronavirus-2019/events-as-they-happen (accessed on 13 May 2020)

12- World health organization . Q\&A on coronaviruses (COVID-19) . available from : https://www.who.int/emergencies/diseases/novel-coronavirus-2019/question-and-answers-hub/qa-detail/q-a-coronaviruses

13- Bao-Liang Zhong, Wei Lu, Hai-Mei Li., et al. , Knowledge, attitudes, and practices towards COVID-19 among Chinese residents during the rapid rise period of the COVID-19 outbreak: a quick online cross-sectional survey. International Journal of Biological Sciences . 2020; 16(10): 1745-1752. doi: 10.7150/ijbs.45221 